\title{
Segmentation of Inter-Neurons in Three Dimensional Brain Imagery
}

\author{
Gervase Tuxworth $^{1,2}$, Adrian Meedeniya ${ }^{2}$ and Michael Blumenstein ${ }^{1}$ \\ ${ }^{1}$ School of Information Communication and Technology, Griffith University, Queensland, \\ Australia. \\ ${ }^{2}$ National Centre for Adult Stem Cell Research, Griffith University, Queensland, Australia
}

\begin{abstract}
Segmentation of neural cells in three dimensional fluorescence microscopy images is a challenging image processing problem. In addition to being important to neurobiologists, accurate segmentation is a vital component of an automated image processing system. Due to the complexity of the data, particularly the extreme irregularity in neural cell shape, generic segmentation techniques do not perform well. This paper presents a novel segmentation technique for segmenting neural cells in three dimensional images. Accuracy rates of over $90 \%$ are reported on a data set of 100 images containing over 130 neural cells and subsequently validated using a novel data set of 64 neurons.
\end{abstract}

\section{Introduction}

Microscopy and image acquisition technology has undergone a recent revolution, generating multidimensional data sets that require significant automation to analyse efficiently. Studies have shown that many neurodegenerative disorders (including Parkinson's disease and Alzheimer's disease) have a significant impact on neuronal structure. The ability to recognize changes in the morphology of neurons together with more subtle changes that occur within the cells with respect to the distribution of cytoplasmic proteins and cell organelles, will allow cell function to be determined. The capacity to analyse the morphology of these cells using unbiased, automated techniques would greatly facilitate advances in the field. In order to analyse the morphology of individual neural cells, we must first segment them from the rest of the image.

Our previous work in cell morphology analysis [1] used manually guided segmentation techniques to conduct experiments on our initial data set, but with increasing data becoming available, there is a need for accurate automated image analysis systems. In addition, manual techniques are subject to human bias, making automated quantitative techniques desirable. However, the automated segmentation of neural cells of the central nervous system presents a major challenge due to inherent features such as irregularity of cell shape and size, and arborising interconnecting cell processes. 
Many existing segmentation techniques in fluorescent microscopy concentrate on the segmentation of morphological structures such as the cell nuclei $[2,3]$, or the complete cell (nucleus and cytoplasm) [4]. Fernandez et al. [2] and Jarkrans [3] both try to detect and separate directly neighbouring cell nuclei. Fernandez et al. propose the detection of dominant or concave points on the binary contour of the region as points where a splitting of the nuclei might be possible. In contrast, Jarkrans uses a contour analysis based on a smoothed chain code, which reflects the curvature of the nucleus contour.

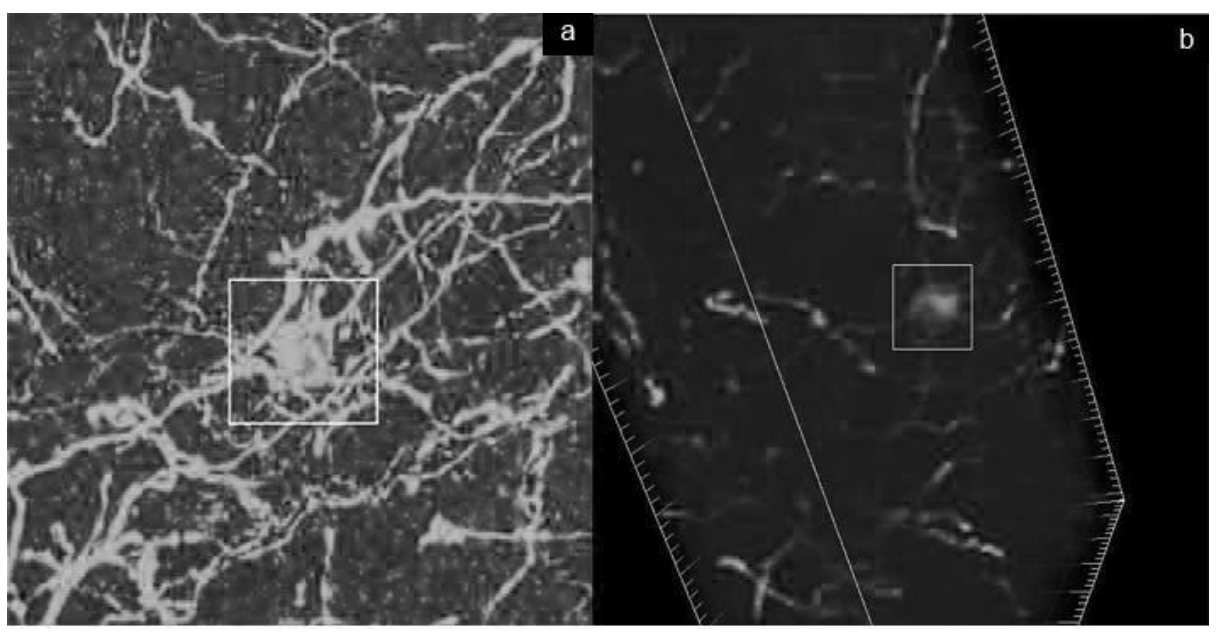

Figure 1. An olfactory bulb neuron in a) 2D MIP Image; b) 3D Volume image

We have developed a method for generating optically resolvable, thick tissue specimens [5]. The cellular and sub-cellular compartments of these specimens are probed using multiple fluorescent probes. These structures may be resolved in three dimensional space using structured illumination and wide-field microscopy ([6], [7]). Thus, we are able to produce large data sets of high resolution three dimensional (3D) images probed with multiple markers [5]. These images contain more information than their two dimensional (2D) counterparts, and present new possibilities for automated analysis. Figure 1 shows a cell that would normally be difficult or impossible to segment in a two dimensional image become a more manageable image processing task when analysed in three dimensions. When considering a three dimensional image, new segmentation techniques are needed to facilitate further processing.

Current three dimensional segmentation techniques can be broken down into edge based techniques and region based techniques. In edge-based approaches such as [8] , the points located on the edges are first identified, followed by edge linking, contour analysis and surface definition. In region-based approaches a number of seed regions are first chosen. These seed regions grow by adding neighbouring points based on a 
compatibility threshold [9]. In the context of fluorescence microscopy, goal seed regions are the cell nuclei or centres where the signal intensity is highest. As the region growing reaches the edges of the cell, the intensity values drop off and therefore don't meet the compatibility threshold.

In [10], Yu et al. propose a seed region finding approach to segmenting tightly packed cell nuclei within 3D confocal images of neurospheres. They focused on a method to find seed regions in the centre of objects of different sizes and then use an "Evolving" Generalized Voronoi Diagram to separate touching nuclei. Another nuclei segmentation algorithm proposed by Long et al [11] uses the 3D watershed method to segment cell nuclei. They combine a foreground contour mask with intensity-based watershed segmentation and post-process the segmentation results to correct over and under segmentation errors.

Our project goals required the entire cell body to be segmented, so these nuclei segmentation techniques may not be suitable. In this paper, we propose a method that involves a 2D slice by slice analysis as per Cai $e t$ al in [12], as well as intensity based analysis as used in [11], and then use contour tracking to merge 2D slices into $3 \mathrm{D}$ neuron masks. We tested this algorithm on 100 images of the olfactory bulb of mice and found we were able to segment cells with high accuracy.

\section{Research Methodology}

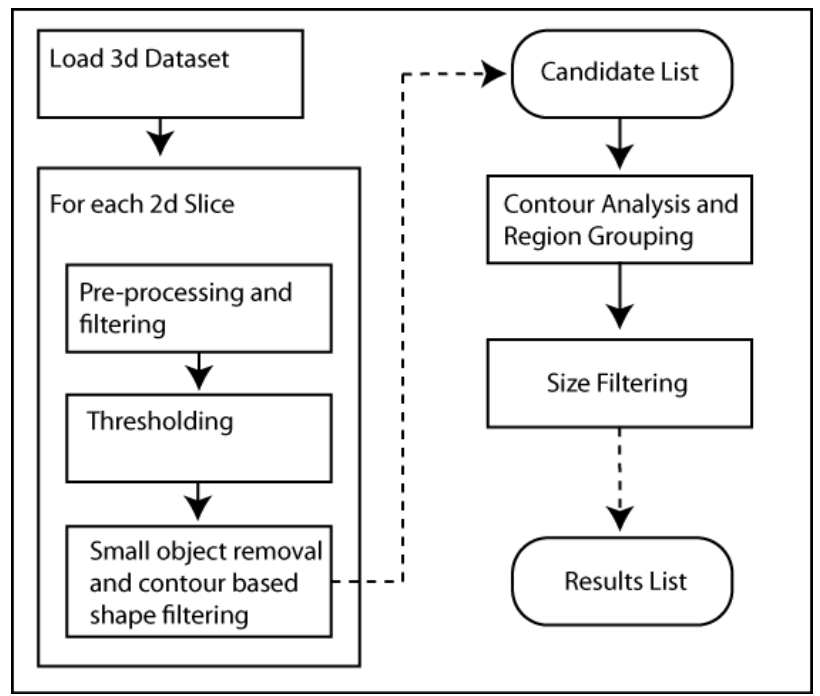

Figure 2. Overview of segmentation process 


\subsection{Pre-Processing}

The 3D image data is loaded as a 'stack' of 2D images. All the individual 2D images ('slices') share the same $\mathrm{x}$ and $\mathrm{y}$ dimensions. The number of images is variable depending on the thickness of the tissue sample, but a typical stack has between 80 and 120 slices, each slice representing $0.25-0.5 \mu \mathrm{m}$ of cell thickness along the $\mathrm{z}$ axis.

\subsection{Slice Analysis}

Each slice has a $3 \times 3 \times 3$ Gaussian filter applied to it to remove noise and is then thresholded to separate the foreground from the background. Similar works ([13], [11]) use Otsu's method [14] for automatically detecting a threshold that separates foreground background. Whilst using an automated thresholding technique does provide more tolerance for intensity variations in the data set, it also has the drawback of looking to discriminate foreground and background data regardless of the image contents. In some images with little 'real' foreground data (an image with no neurons in it), an automatic thresholding technique is more likely to produce false positive objects. Both methods will be used in experimentation.

Once thresholded, each slice then has a series of morphological operations performed on it to remove irrelevant or insignificant objects. Firstly, the slices are filtered again to remove any noise or stray pixels created in the thresholding process. After the slices have been filtered, morphological opening (erosion followed by dilation) is performed to remove small objects followed by morphological closing (dilation followed by erosion) to fill in holes inside foreground objects.

Once the objects have been separated, we identify potential neuron cross sections by eliminating small objects and computing a contour roundness metric. After estimating the perimeter and area of each object in the scene, an isoperimetric value is calculated using the formula:

$$
\frac{4 \pi \text { area }}{\text { perimeter }^{2}}
$$

If the objects isoperimetric value is below a preset threshold, the object is discarded as a candidate; else it is added to the candidate list. With an appropriate threshold, this filtering allows our technique to remove foreground objects like thick axon trunks, whose cross sections are typically more rectangular shaped than neural cell bodies.

\subsection{Neuron Segmentation}

Once each slice has been individually analysed and objects placed on the candidate list, the objects on the list need to be grouped to create 3D masks. Assuming a conservative minimum cell thickness of 3 microns, an object must be present in at least 
12 slices (at $0.25 \mu \mathrm{m}$ slice thickness) to be considered. We discard any neurons touching image boundaries as they may not contain complete neurons. Objects that overlap in the $\mathrm{x}$ and $\mathrm{y}$ planes on adjacent images are examined for contour similarity. A high rate of change of the contour of the object over a series of slices can be used to identify overlapping or touching objects, or identify slices with noisy data around the cell. By creating a rate of change threshold, objects that have irregularities in their contours between Z-slices can be discarded.

Once the neurons have been identified, they can be segmented in one of two ways. A bounding box can be created using the maximum detected radius of any slice to identify where a neuron is located. This is useful for cell counting applications, but does not suit applications where the morphology of the cell is important. Instead, a three dimensional matrix can be constructed as a mask over the original image, with the detected cell cross-section in each slice used to create a series of two dimensional masks. This extracts the morphology of the cell for further analysis.

\subsection{Algorithm Pseudo-code}

Psuedo-code for the segmentation technique can be seen below.

\section{Segmentation Algorithm}

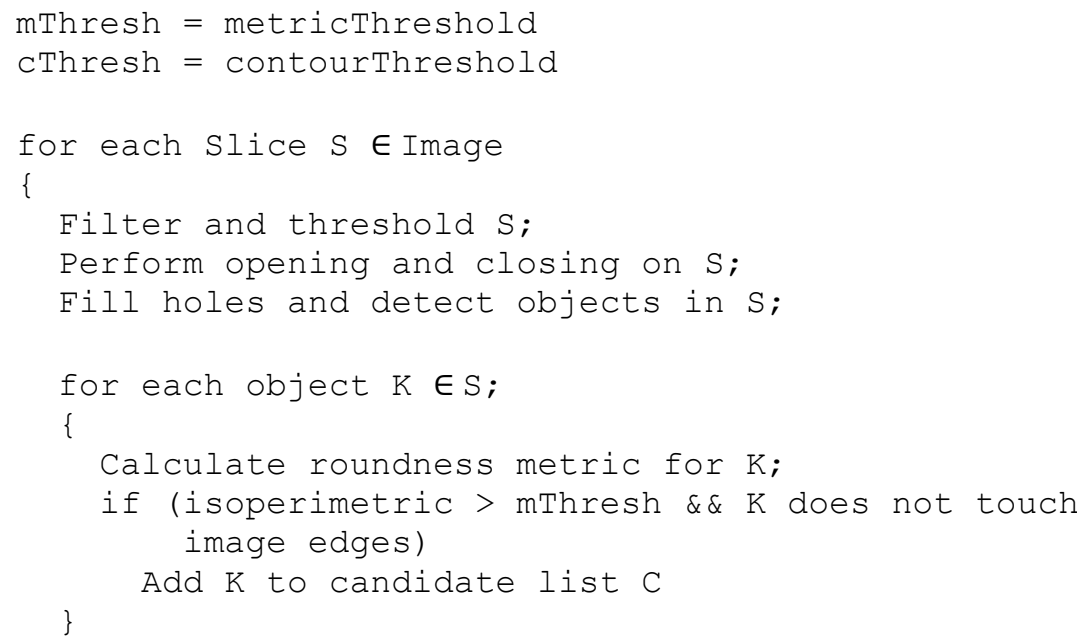




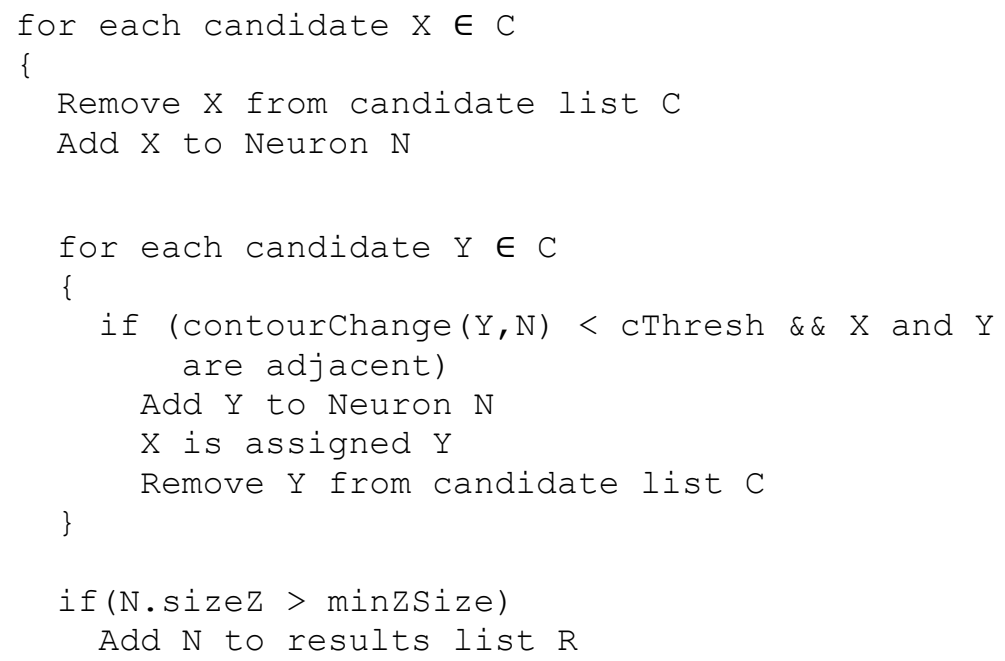

\section{Experimental Results, Analysis and Discussion}

To develop our proposed segmentation technique, 100 images of olfactory bulb neurons were obtained. Tissue specimens were harvested from laboratory mice, embedded in PEG using the protocol detailed in [5] and sectioned coronally at a thickness of $40 \mu \mathrm{m}$. Fifty images were taken of olfactory neurons from wild type laboratory mice and another 50 were taken from mice with a focal deletion of the gene sequence coding for the neurotrophic factor neurturin.

Images were taken at 630x magnification using a Zeiss AxioImager Z1 equipped with an Apotome system to create Z-stacks. These images were manually analysed by neurobiologists and found to contain 134 cells. We tested our algorithm using several different parameters for threshold, minimum object size and isoperimetric threshold.

Table 1. Results using manual threshold values

\begin{tabular}{lllc}
\hline $\begin{array}{l}\text { Minimum } \\
\text { Object Size }\end{array}$ & $\begin{array}{l}\text { Isoperimetric } \\
\text { Threshold }\end{array}$ & Threshold & Accuracy \\
\hline 2500 & 0.7 & 0.2 & $74 \%$ \\
$\mathbf{2 5 0 0}$ & $\mathbf{0 . 7}$ & $\mathbf{0 . 3}$ & $\mathbf{8 8 \%}$ \\
2500 & 0.7 & 0.4 & $80 \%$ \\
4000 & 0.7 & 0.2 & $77 \%$ \\
4000 & 0.7 & 0.3 & $83 \%$ \\
4000 & 0.7 & 0.4 & $72 \%$ \\
\hline
\end{tabular}


The highest accuracy was obtained using a fairly low threshold (0.3), with 123 detected cells and 5 miss-classifications, resulting in an accuracy of 118/134 (88\%). Lowering the threshold to 0.2 increased the number of false positives, while any higher resulted in an increased number of missed cells. We then used Otsu's method to automatically calculate the threshold for the dataset.

Table 2. Otsu's thresholding vs. manual thresholding

\begin{tabular}{lccc}
\hline Threshold & $\begin{array}{l}\text { False } \\
\text { Positives }\end{array}$ & $\begin{array}{l}\text { Missed } \\
\text { Cells }\end{array}$ & Accuracy \\
\hline 0.3 & 5 & 11 & $88.1 \%$ \\
Otsu & $\mathbf{7}$ & $\mathbf{8}$ & $\mathbf{8 8 . 8 \%}$ \\
\hline
\end{tabular}

The use of Otsu's thresholding technique yielded 126 detected cells, but 7 missclassifications giving an overall accuracy of $88.8 \%$. As expected, Otsu's technique created more foreground data than a preset manual value and resulted in an increase in false positives. To attempt to reduce the number of false positives, we tested Otsu's technique with higher post processing thresholds for object size and isoperimetric values.

Table 3. Adjusting algorithm parameters with Otsu's thresholding technique

\begin{tabular}{lcccc}
\hline $\begin{array}{l}\text { Minimum } \\
\text { Object Size }\end{array}$ & $\begin{array}{l}\text { Isoperimetric } \\
\text { Threshold }\end{array}$ & $\begin{array}{l}\text { False } \\
\text { Positives }\end{array}$ & $\begin{array}{l}\text { Missed } \\
\text { Cells }\end{array}$ & Accuracy \\
\hline 2500 & 0.7 & 7 & 8 & $88.8 \%$ \\
2500 & 0.75 & 6 & 17 & $82.8 \%$ \\
$\mathbf{4 0 0 0}$ & $\mathbf{0 . 7}$ & $\mathbf{2}$ & $\mathbf{1 0}$ & $\mathbf{9 1 . 1 \%}$ \\
4000 & 0.75 & 2 & 19 & $84.3 \%$ \\
\hline
\end{tabular}

By increasing the post-processing thresholds we are able to eliminate nearly all of the false positives without significantly compromising the correctly labelled cells. This is particularly useful in our application where we can generate large data sets, so missed cells will have little impact, but a large number of false positives could cause inaccuracies in the analysis of cell morphologies.

To test our results, a separate validation data set of 30 images was obtained and segmented using the parameters that produced the best results on our original "development" dataset. 
Table 4. Accuracy of algorithm on validation dataset.

Minimum object size 4000, Isoperimetric threshold 0.7

\begin{tabular}{lccc}
\hline Dataset & $\begin{array}{l}\text { False } \\
\text { Positives }\end{array}$ & $\begin{array}{l}\text { Missed } \\
\text { Cells }\end{array}$ & Accuracy \\
\hline Original (100 images, 134 cells) & 2 & 10 & $91.1 \%$ \\
Validation (30 images, 64 cells) & 2 & 5 & $89.1 \%$ \\
\hline
\end{tabular}

The validation images contained a high density of neurons per image in comparison to the development data set. Despite the increased density of cells in the validation set, our segmentation technique was able to segment cells with an accuracy of $89.1 \%$. The number of false positives was relatively low, and only 5 out of the 64 cells were not recognized.

Our overall accuracy of $91.1 \%$ and $89.1 \%$ is comparable to other segmentation techniques ([11], [12]) developed for related 3D microscopic image analysis tasks. A closer inspection of the results also showed that $7 / 10(70 \%)$ of the missed cells in the original data set occurred in just $4(4 \%)$ of the images where there is a low signal to noise ratio either due to a weak fluorescent signal or a high non-specific background signal. As our aim is to completely automate the image analysis process, the weak images are not manually removed from the dataset, however an extension to our work will involve the automated identification of poor images and their removal from the dataset to prevent biasing of the study outcomes.

\section{Conclusions and Future Research}

In this paper we have presented a technique for segmenting neurons in 3-Dimensional images that combines both edge based and region growing techniques. This algorithm requires very little manual input and is able to automatically detect seed points in complex scenes. Using contour analysis we can accurately detect objects over a series of slices and determine whether or not they are a neuron. The technique was validated using a novel data set of greater complexity and demonstrated to work accurately. This work provides a critical component for future automated image analysis. Using the segmented neuron data, we can develop techniques to automatically analyse neuron morphology and population numbers to aid neurobiological and other cell biological research.

\section{Acknowledgements}

The authors would like to acknowledge the invaluable assistance of the members of the Transplant Therapy and Neuro Anatomy group at the National Centre for Adult Stem Cell Research, Griffith University. In particular, we would like to thank Brenton Cavanagh and Maria Nguyen for tissue preparation and imaging. 


\section{References}

[1] A. Alavi, Cavanagh, B., Tuxworth, G., Meedeniya, A., Mackay-Sim, A. and Blumenstein, M., "Automated classification of dopaminergic neurons in the rodent brain," presented at the International Joint Conference on Neural Networks, Atlanta, 2009.

[2] G. Fernandez, Zyrd, J.P. and Kunt, M. , "A New Plant Cell Image Segmentation Algorithm," presented at the ICIAP, 1995.

[3] T. Jarkrans, "Algorithms for Cell Image Analysis in Cytology and Pathology," Faculty of Science and Technology, Uppsala University, Sweden, 1996.

[4] J. Byriel, "Neuro-Fuzzy Classification of Cells in Cervical Smears," Technical University of Denmark, Lyngby, 1999.

[5] M. N. Nguyen, Cavanagh, B., Davenport, T., Norazit, A., Meedeniya, A., "Tissue processing for epifluoresence microscopy," in Microscopy: Science, Technology, Applications and Education. vol. 4, A. Méndez-Vilas, Diaz, J., Ed., ed Badajoz, Spain: Formatex, 2010.

[6] L. H. Schaefer, Schuster, D. and Herz, H., "Generalized approach for accelerated maximum likelihood based image restoration applied to three-dimensional fluorescence microscopy," Journal of Microscopy, vol. 204, pp. 99-107, 2001.

[7] J. M. Murray, Appleton, P.L., Swedlow, J.R. and Waters, J.C., "Evaluating performance in three-dimensional fluorescence Microscopy," Journal of Microscopy, vol. 228, pp. 390-405, 2007.

[8] R. Benlamri, "Range image segmentation of scenes with occluded curved objects," Pattern Recognition Letters, vol. 21, pp. 1051 - 1060, 2000.

[9] X. Jiang, Bunke, H., Meier, U., "High level feature based range image segmentation," Image and Vision Computation, vol. 18, pp. 817 - 822, 2000.

[10] W. Yu, et al., "Segmentation of Neural Stem/Progenitor Cells Nuclei within 3-D Neurospheres," in Advances in Visual Computing. vol. 5875, G. Bebis, et al., Eds., ed: Springer Berlin / Heidelberg, 2009, pp. 531-543.

[11] F. Long, et al., "Automatic Segmentation of Nuclei in 3D Microscopy Images of C.Elegans," in Biomedical Imaging: From Nano to Macro, 2007. ISBI 2007. 4th IEEE International Symposium on, 2007, pp. 536-539.

[12] H. Cai, et al., "Using nonlinear diffusion and mean shift to detect and connect crosssections of axons in 3D optical microscopy images," Medical Image Analysis, vol. $12,2008$.

[13] W. Yu, et al., "Segmentation of Neural Stem/Progenitor Cells Nuclei within 3-D Neurospheres," in Advances in Visual Computing, ed, 2009, pp. 531-543.

[14] N. Otsu, "A Threshold Selection Method from Gray-Level Histograms," Systems, Man and Cybernetics, IEEE Transactions on, vol. 9, pp. 62-66, 1979. 